Un paso más: evaluación del impacto de género. Guía para evaluar el impacto de género de los proyectos de cooperación para el desarrollo. Murguialday, Clara* y Vázquez, Norma**. (2005). Barcelona: Cooperacció. (63 páginas).

\title{
Paso adelante, paso atrás: Evaluando una guía de evaluación
}

\section{A step forward, a step backward: Evaluating an evaluation guide}

\author{
Reseñada por Gabriela Castellanos Llanos ${ }^{* * *}$ \\ Centro de Investigaciones y Estudios de Género, Mujer y Sociedad, \\ Universidad del Valle, Cali, Colombia.
}

Recibido: 21 de septiembre de 2018. Aprobado: 29 de enero de 2018. DOI: 10.25100/lamanzanadeladiscordia.v13i1.6717

Reseña

Palabras clave: Evaluación de proyectos; perspectiva de género; empoderamiento; equidad de género.

Keywords: Project evaluation; gender perspective; empowerment; gender equity.

\footnotetext{
*Licenciada en Economía, ha realizado cursos de posgrado en Métodos de Investigación sobre Mujeres (CIPAF, Universidad de Sto. Domingo) y en Desarrollo y Cooperación Internacional (HEGOA). Entre 1980 y 1998 trabajó en Nicaragua, República Dominicana, Uruguay y El Salvador como investigadora, formadora y consultora sobre la incorporación de las mujeres a los programas de desarrollo para diferentes organizaciones. Ha investigado y publicado sobre temas relativos a las mujeres (organización, participación sindical y política, impacto de la guerra, construcción de la ciudadanía en la posguerra, etc.). Imparte clases sobre Género y Desarrollo en varios Masters y colabora con la Coordinadora de ONGD del País Vasco. Ha elaborado diversos conceptos relativos a las cuestiones de género y la problemática de las mujeres.

**Docente en varias escuelas de empoderamiento de Bizkaia y de Gipuzkoa, en el país vasco, en algunas de ellas desde sus inicios. También colabora con ellas en el planteamiento, sobre todo teórico y filosófico, de sus bases y su dinámica de funcionamiento.

***Profesora de la Línea en Estudios de Género del Doctorado en Humanidades, Universidad del Valle. Ensayista, poeta y novelista. Ph. D. en Análisis del Discurso, Universidad de la Florida, 1990. Directora del Grupo de Investigación Género, Literatura y Discurso de la Universidad del Valle. Cofundadora y directora hasta 2015 del Centro de Investigaciones y Estudios de Género, Mujer y Sociedad de la Universidad del Valle. Sus obras más recientes son: Rebelión contra el olvido. Mujeres escriben sobre textos de mujeres (2016; compiladora con Mery Cruz); Jalisco pierde en Cali, novela (2015); Bocetos para una autobiografía, poemario (2014). Correo electrónico: gabicastellanos1@gmail.com.
} 
Fuente: http://www.mujeresenred.net/IMG/pdf/ Un_paso_mas_evaluacion_del_impacto_de_genero.pdf.

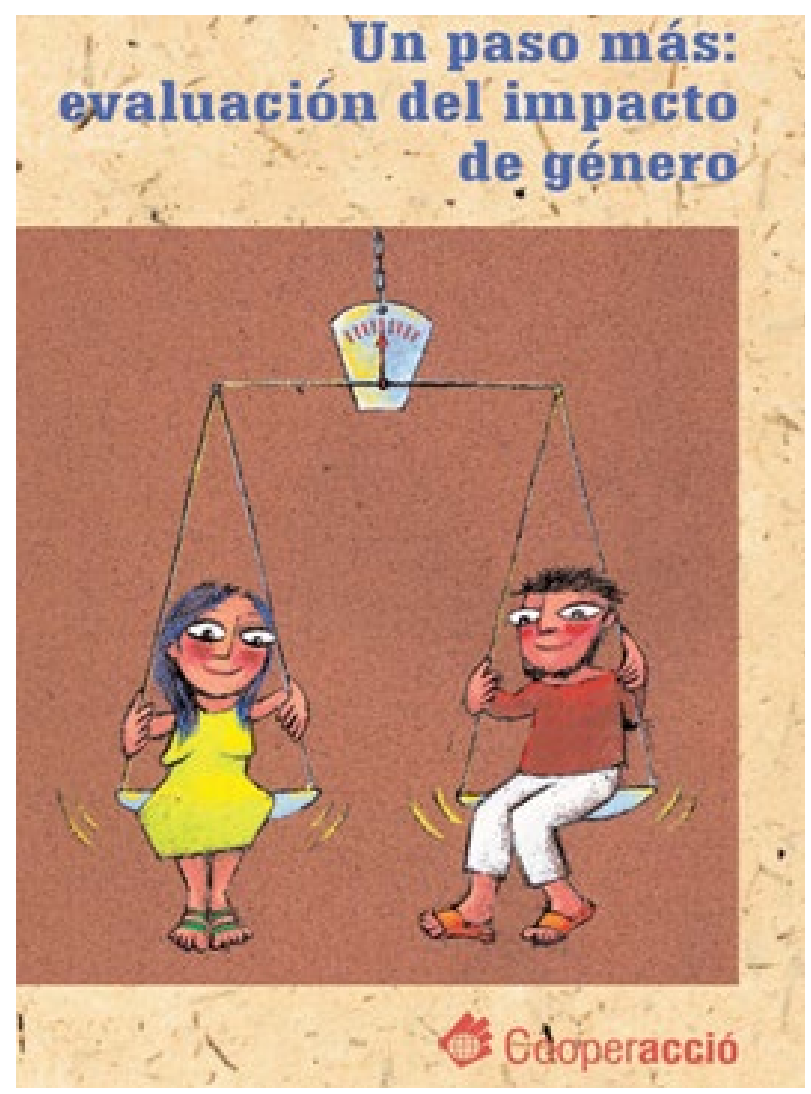

Figura 1. Carátula de la guía "Un paso más: evaluación del impacto de género" de Clara Murguialday y Norma Vásquez.

Este folleto de 65 páginas se propone brindar una serie de concepciones y pistas para mejorar "la calidad de la cooperación internacional desarrollando un instrumento que a las organizaciones que trabajamos en este campo nos permita evaluar los resultados de nuestra labor" (Murguialday y Vásquez, 2005 , p. 1). Es un propósito por demás loable, pues se trata de conocer más a fondo los efectos de un trabajo llevado a cabo para mejorar la situación y por ende la vida de determinadas personas, a fin de lograr mejores resultados en proyectos futuros.

Mi interés por reseñar esta guía se debe a que he encontrado referencia a ella en varios trabajos académicos recientes, y a que deseo entrar en diálogo con este texto en torno a algunas concepciones que allí aparecen. Es fundamentalmente una de las definiciones que hacen las autoras, la de relaciones de género, la que he encontrado en algunas tesis y la que me propongo debatir. Pero antes de entrar en materia, debo contextualizar el texto en los trabajos sobre este tipo de evaluación, así como describir su contenido y sus alcances.

Sobre este tema de la evaluación de proyectos con perspectiva de género existen múltiples publicaciones en América Latina, casi tantas como existen ministerios o dependencias encargadas de desarrollar programas de género en la región. ${ }^{1}$ Existen también otros textos menos generales, con propósitos más específicos, como la Guía de evaluación de programas y proyectos con perspectiva de género, derechos humanos e interculturalidad, de ONU Mujeres (2014), que se distingue por integrar los derechos humanos y la interculturalidad al trabajo con género; o GEM: Gender Evaluation Methodology, de la Asociación para el Progreso de las Comunicaciones (2009), que está enfocada a "incorporar un análisis de género en las evaluaciones de iniciativas que utilizan las tecnologías de la información y la comunicación (TIC) para el cambio social".

Encontramos asimismo una enorme cantidad de reflexiones de tipo académico sobre el tema de la evaluación con perspectiva de género; un ensayo que hace referencia a muchos documentos de este tipo es el trabajo de Julia Espinosa, La evaluación sensible al género: Una herramienta para mejorar la calidad de la ayuda (2010).

Veamos entonces los contenidos de esta guía de Murguialday y Vásquez (2005). En el primer capítulo, titulado "Lo que necesitamos: evaluar los cambios generados", se define la evaluación como "un proceso que se utiliza para medir, analizar y valorar, las consecuencias de una intervención ...en la población con la que se ha llevado a cabo," a fin de "constatar si los resultados obtenidos han sido útiles para mejorar la vida de las personas" (p. 7); asimismo, se justifica la necesidad de la evaluación. Las autoras definen el "impacto de género" como "el criterio de evaluación que mide las consecuencias positivas y negativas que ha tenido una intervención de desarrollo en las relaciones de género, es decir,

${ }^{1}$ No debe esperarse en este tipo de publicaciones que se ventile el tema de la crítica al concepto de desarrollo, ni que se haga referencia al "posdesarrollo". No se hace en ninguna de las guías que he revisado, ni ocurre tampoco en esta que aquí reseño. 
las que se establecen entre mujeres y hombres" ( $\mathrm{p}$. 8). Se analizan proyectos específicos desarrollados en Guatemala y Nicaragua respectivamente, y se distingue entre evaluación ciega al género, evaluación con enfoque de género y evaluación del impacto de género. En este último tipo de evaluación se indaga sobre cómo ha cambiado la actitud de mujeres y hombres sobre la conveniencia de atender las necesidades de las mujeres, y si se ha transformado o no la relación de pareja.

El segundo capítulo, denominado "Lo que entendemos por "cambiar las relaciones de género", distingue entre "relaciones de género" y "situación de las mujeres" y se define el término "relaciones de género" (más adelante discutiremos esta definición). Se analiza allí una serie de citas tomadas de testimonios "de mujeres que han sido beneficiarias de microcréditos para iniciar proyectos productivos en Nicaragua" (p. 13), y de otras participantes de un proyecto en Guatemala, enfatizando la falta de análisis sobre las relaciones de género y el "énfasis equivocado en el colectivo femenino como único responsable de su situación" que en esos testimonios se advierte (p. 15). Se analiza también un ejercicio realizado en un proyecto, donde se utilizó como instrumento una agenda de las actividades del día de hombres y mujeres, y se constata que nunca se cuestionan las razones para, por ejemplo, el mayor descanso de los hombres frente al de las mujeres. Se tiende allí, según las autoras, más a describir que a dilucidar las relaciones de poder entre los géneros. Asimismo, la falta de consideración sobre las opiniones y actitudes de los hombres, la "ausencia de los hombres" en los proyectos, a menudo conduce a que se llegue a conclusiones equivocadas.

El tercer capítulo "Lo que no buscas bien, no ves': herramientas para evaluar el impacto de género", enfatiza la necesidad de contar con una línea de base, que las autoras denominan "información 'de partida' sobre las relaciones de género", a fin de contrastar la situación y las relaciones anteriores con las que se consiguen mediante el desarrollo de los proyectos. Se afirma también la importancia de "prever tanto los efectos positivos como los negativos que toda intervención de desarrollo puede acarrear" (p. 24), así como de plantear hipótesis que se puedan comprobar en el trayecto del trabajo, a la vez que tomar en cuenta indicadores de género. Partiendo del enfoque GED (Género En el Desarrollo), las autoras presentan un esquema de preguntas, categorías e instrumentos a utilizar, desarrollado por Birgit Kerstan (1996). Estos instrumentos incluyen: perfil de actividades, matriz de acceso y control de recursos y beneficios, cuadro de necesidades prácticas e intereses estratégicos, perfil de la posición de las mujeres respecto a los hombres, y factores influyentes. Adaptando y resumiendo los aportes de muchos otros autores, pasan luego a discutir cada uno de estos instrumentos, lo cual constituye probablemente la sección más útil de la guía.

En el cuarto capítulo "Lo que perseguimos: empoderamiento y equidad", desglosan "el proceso de empoderarse", y definen una serie de criterios para contestar la pregunta "¿Cómo sabemos que el impacto de género ha sido positivo?” (p. 43). Aquí elaboran su propio esquema que detalla los factores de empoderamiento subjetivo de las mujeres, en lo personal, en sus relaciones con la pareja y la familia, y en relación con el ámbito público. También apelan a otros autores para analizar cómo puede medirse el empoderamiento subjetivo, y cómo se define y se determina el empoderamiento económico. Finalmente distinguen varias formas de poder: poder sobre, o dominación; poder interno, que nos remite a los cambios subjetivos; poder con, que tiene que ver con las relaciones con otros y otras, con colectivos; y poder para, que se encamina a determinar si se ha logrado "transformar las estructuras que sostienen la desigualdad de género" (p. 56). Cada una de estas categorías es discutida a fondo, y de manera que parece muy útil para quienes necesitan realizar evaluaciones de proyectos encaminados a empoderar a las mujeres. Finalmente, esbozan un horizonte para la transformación deseada: "el desarrollo humano con equidad de género” (pp. 61-62).

En general, Murguialday y Vásquez dan muestras de haber realizado una serie de pesquisas que las llevan a cubrir los autores más sobresalientes en el tema, y logran describir las ideas y procedimientos recomendados de manera clara y sucinta. Al recopilar los aportes de muchos estudios y propuestas, y al ponerlos al servicio de mejorar las evaluaciones de los proyectos que buscan integrar la perspectiva de género al trabajo en pro del desarrollo, las au- 
toras hacen una contribución real. Y, lo que es aún más importante, su énfasis en que los proyectos y programas no se queden en la mera descripción de diferencias en los roles de género, sino que profundicen en la evaluación del impacto real que se ha logrado en las relaciones de género puede resultar muy útil para mejorar estos proyectos. Todo lo anterior significa una serie de pasos adelante en el camino de mejorar los proyectos que se proponen contribuir al empoderamiento de las mujeres. Pero (siempre hay un pero) este texto enturbia un tanto las aguas al basarse extensamente en una definición pobre de "relaciones de género". En ese sentido, puede considerarse un paso atrás.

La guía define las relaciones de género como "la manera en que mujeres y hombres se relacionan generando posiciones de desigualdad en las que lo femenino y la mayoría de las mujeres estarán en el polo subordinado en tanto que lo masculino y la mayoría de los hombres conforman el polo dominante" (p. 16). Veamos, en primer lugar, varios aciertos de las autoras al discutir este concepto.

Como ya se dijo, las autoras distinguen con justeza "relaciones de género" de una mera "descripción de la situación de las mujeres" (p. 13). Asimismo, explican por qué el concepto no es igual a "roles diferentes de mujeres y hombres" (p. 15), sino que va mucho más allá, pues nos remite a "relaciones de poder, desigualdad, jerarquías" (p. 16). En tercer lugar, al aludir no sólo a hombres y mujeres sino también a lo femenino y lo masculino están incorporando en cierta medida el ámbito cultural en su definición.

Sin embargo, dicha definición presenta una serie de deficiencias. Al reducir las relaciones de género a las que se dan entre sujetos hombres y mujeres, aún si se consideran en su dimensión cultural, se reduce significativamente el alcance del concepto, pues se lo personaliza, como si se tratara solo de relaciones interpersonales entre individuos. Como veremos, las relaciones de género son mucho más amplias y significativas, pues incluyen tanto la dimensión simbólica como relaciones mucho más diversas, en lo social, económico y político y no solo en lo personal.

Además, las autoras nunca definen lo que entienden por "mujeres" o por "hombres", por lo cual no sabemos si toman en cuenta a los sujetos “transgenerizados". A partir de allí, es fácil caer en una reducción de "mujer" y "hombre" a lo genital, desconociendo las críticas feministas a estos reduccionismos (Butler, 2001). La definición ofrecida tampoco nos permite tomar en cuenta las relaciones entre varones y entre mujeres como relaciones de género. Esto es una grave deficiencia, y no sólo en lo que atañe a la sexualidad; las relaciones entre mujeres heterosexuales y entre hombres heterosexuales están también fuertemente condicionadas por todo el universo de las relaciones de género.

En segundo lugar, al apelar al concepto de mayorías ("mayoría de las mujeres", "mayoría de los hombres") las autoras dan a su definición una base estadística, como si la dominación y la subordinación fueran asunto de números. En este sentido las autoras, quizá por estar centradas en el mundo de las estadísticas, de los análisis económicos sobre el desarrollo, parecen descuidar las reflexiones filosóficas, sociológicas, antropológicas, que hemos logrado las feministas, que inclusive han entrado a formar parte de las problemáticas estudiadas no sólo por las feministas, sino también por la corriente principal de muchas disciplinas.

Me parece evidente que la labor de proponer y apoyar la equidad de género no consiste en aumentar las cantidades de mujeres libres de dominación o disminuir las cantidades de hombres dominadores, sino de lograr una profunda transformación social y cultural. Por lo demás, ¿acaso existe una minoría de mujeres que no están subordinadas debido al género, que están exentas de la dominación masculina? ¿Acaso podemos encontrar una minoría de hombres que no están en posición dominante en cuanto al género, es decir, que por algún motivo están, o bien dominados por razones de género, o por fuera de este tipo de relaciones? La jerarquía social que ubica a los hombres en el polo dominante y a las mujeres en el dominado en cuanto al género es de aplicación universal.

El hecho de que haya algunas mujeres con poder social, económico e inclusive político significativamente mayor que el de algunos hombres no es cuestión de cantidades de individuos, sino de lo que lo que Nancy Fraser (1997) famosamente llamó "múltiples diferencias que se intersectan", o lo que, 
con base en la metáfora de Fraser sobre líneas en intersección donde se cruzan distintos parámetros de dominación, recientemente se ha dado en llamar "interseccionalidad". Se trata de tomar en cuenta cómo se entrecruzan formas de dominación con base en el género con otras que se basan en la clase social, la "raza", la etnia, la sexualidad, entre otras. En esas intersecciones, encontramos sujetas mucho más poderosas por razones económicas y políticas que ciertos hombres. Pero en igualdad de condiciones, la dominación de género significa que los hombres estarán en últimas en posición de dominio, las mujeres siempre en posición de desventaja, a pesar de sus esfuerzos y sus resistencias.

Finalmente, las autoras en ningún momento mencionan la violencia simbólica: los múltiples discursos y prácticas sociales que conducen a que la dominación no sólo sea aceptada por los dominados y las dominadas como justa y natural, sino que esté inscrita en los mismos cuerpos.

Por todo lo anterior, necesitamos una definición de "relaciones de género" que subsane las deficiencias que venimos discutiendo. Se requiere, no sólo porque los trabajos en pro del desarrollo deben basarse en concepciones bien cimentadas, sino porque textos como el de Murguialday y Vásquez tienen repercusión no sólo en el ámbito de dichos trabajos, sino en un círculo más amplio que influye en el mundo académico.

Por eso ofrezco mi propia definición: Relaciones de género alude al conjunto de diferencias, intercambios discursivos, tensiones y conflictos entre sujetos de cualquier género, que se basa en las diferencias culturales, económicas, políticas y legales entre hombres y mujeres por su pertenencia a estas dos categorías sociales, y en las ideologías y discursos sobre estas diferencias, tensiones y conflictos, así como en las prácticas sociales relacionadas. Entre estos discursos y prácticas encontramos los que Bourdieu (2000) denominó "violencia simbólica", que tiene que ver con aquellos que tienen por efecto convencer a los dominados y las dominadas de la justeza e inevitabilidad de la dominación que padecen, habituándolas al sometimiento, a la vez que los dominadores se convencen de su derecho a la domi- nación; estas convicciones y hábitos se inscriben en los cuerpos mismos de ambos grupos.

Además, aunque la base ideológica y social de las relaciones de género la constituyen las diferencias culturales y sociales entre hombres y mujeres, las relaciones de género se extienden a las relaciones de varones entre sí y de mujeres entre sí, y a las relaciones que atañen a las diversidades sexuales y de género, cuando todas éstas tienen raíces en dichas diferencias culturales y sociales.

Por otra parte, la consideración y el análisis de las relaciones de género deben tomar siempre en cuenta la interseccionalidad, es decir, la incidencia de otras formas de jerarquización social, por razones de clase, raza, etnia, sexualidad, etc. que se entrecruzan con las primeras.

En suma, esta definición nos invita a abandonar la reducción de las relaciones de género a lo interpersonal, a tomar en cuenta las dimensiones más amplias de las relaciones sociales, así como la interseccionalidad y las relaciones intra-género, entre varones y entre mujeres, y a revisar nuestra definición de los conceptos de hombre y de mujer.

\section{Referencias bibliográficas}

Bourdieu, Pierre. (2000). La dominación masculina (Joaquín Jordá, Trad.). Barcelona: Anagrama. (Obra original publicada en 1998).

Butler, Judith. (2001). El género en disputa. El feminismo y la subversión de la identidad (María Mufloz, Trad.). México: Paidós. (Obra original publicada en 1990).

Espinosa, Julia. (2010). La evaluación sensible al género: Una herramienta para mejorar la calidad de la ayuda. En XIV Encuentro de Latinoamericanistas. Congreso llevado a cabo en Santiago de Compostela, España. Recuperado de https://halshs.archives-ouvertes.fr/halshs-00532615/document.

Fraser, Nancy. (1997). Iustitia interrupta: reflexiones críticas desde la posición "postsocialista" (Magdalena Holguín e Isabel Jaramillo, Trads.). Bogotá: Siglo del Hombre Editores.

Murguialday, Clara y Vásquez, Norma. (2005). Un paso más: evaluación del impacto de género. Guía para evaluar el impacto de género de los proyectos de cooperación para el desarrollo. Barcelona: Cooperacció. Recuperado de http://www.mujeresenred.net/IMG/pdf/ Un_paso_mas_evaluacion_del_impacto_de_genero.pdf. 